\section{Pathophysiology of Haemostasis and Thrombosis}

Pathophysiol Haemost Thromb 2005;34:80-90

DOI: $\underline{10.1159 / 000089929}$

\title{
Stochastic Modeling of Blood Coagulation Initiation
}

\author{
Ken Lo ${ }^{1}$ William S. Denney ${ }^{1}$ Scott L. Diamond \\ Institute for Medicine and Engineering, Department of Chemical and Biomolecular Engineering, \\ University of Pennsylvania, 1024 Vagelos Research Laboratories, Philadelphia, Pa., USA
}

\section{Key Words}

Fluorescence $\cdot$ Thrombin $\cdot$ Kinetics $\cdot$ Coagulation $\cdot$ Monte Carlo

\begin{abstract}
A kinetic Monte Carlo simulation was developed using the deterministic reaction network developed by the Mann laboratory for tissue-factor (TF)-initiated blood coagulation. The model predicted thrombin dynamics in recalcified whole blood (3-fold diluted) pretreated with convulxin (platelet GPVI activator) and picomolar levels of TF (0-14 pM). The model did not accurately predict coagulation times at low TF $(0-0.7 \mathrm{pM})$. The simulation revealed that $\sim 0.2 \mathrm{pM}$ TF was the critical concentration to cause $50 \%$ of reactions containing 3 -fold diluted whole blood to reach a clotting threshold of $0.05 \mathrm{U} / \mathrm{ml}$ thrombin by $1 \mathrm{~h}$. Simulations of $1 \mathrm{nl}$ of blood (5 pM TF) revealed small stochastic variations in thrombin initiation time, while $16.6 \mathrm{pl}$ simulations were highly stochastic at this level of TF (50 molecules/16.6 pl). Further experiment and simulation will require evaluation of mechanisms of coagulation kinetics at subpicomolar levels of TF.
\end{abstract}

Equal contributions.

\section{Introduction}

Kinetic models of tissue-factor (TF)-triggered blood clotting are quite advanced and are beginning to incorporate aspects of intracellular metabolism, hemodynamic regulation of transport physics, and receptor-mediated adhesion [1-3], which are unique to blood as a proteomic system. Most modeling efforts concentrate on using deterministic reaction rate equations to predict what is occurring in a well-mixed volume of blood [4]. Several groups $[1,5]$ have explored the significance of platelet surface to blood coagulation, so efforts towards understanding the nature of cell-protein interaction in blood coagulation are well underway. The effects of hemodynamics within blood coagulation account for convection and flow-enhanced diffusive transport (dispersion) [1-3].

Special numerical methods have been required [6], since blood coagulation is a stiff system, with several reactions acting much faster than others. Much of this work has been done deterministically, utilizing stiff equation solvers such as the Livermore Solver for Ordinary Differential Equations. A stochastic Monte Carlo (MC) method first proposed by Gillespie [7] has proven especially useful for blood systems that have hundreds to thousands of species undergoing $>10^{5}$ reactions and concentrations ranging from one molecule per $\mathrm{nl}$ to micro-

\begin{tabular}{ll}
\hline KARGER & ( ) 2005 S. Karger AG, Basel \\
Fax +4161306 1234-8832/05/0343-0080\$22.00/0 \\
$\begin{array}{l}\text { E-Mail karger@karger.ch } \\
\text { www.karger.com }\end{array}$ & $\begin{array}{l}\text { Accessible online at: } \\
\text { www.karger.com/pht }\end{array}$
\end{tabular}


molar levels of concentration [3]. This method can be more computationally expensive, but provides exact dynamics of low-concentration compounds. Blood coagulation simulation is dependent on accurate simulation of low concentrations, as initially factor Xa and thrombin are both formed slowly at very low concentrations. Also, it has been observed that blood coagulation times can display experimental variability at low TF concentrations [8]. MC allows prediction of individual clotting scenarios as well as the standard deviation due to stochastic effects at low concentrations. Deterministic models produce a single result, representing experimental averages.

As a reactive biological fluid, blood and its fractions are fully amenable to liquid handling and fluorogenic substrate probes for real-time monitoring of protease function [9-12], including microarray reactions with nanoliter volumes [13-15]. This allows large data sets to be generated for a single blood sample [8]. In recent years, the regulatory complexity and triggering mechanisms in blood treated with exogeneously added TF have been explored and refined [16], without the confounding effects of contact activation [17, 18]. Presently, high throughput techniques are starting to be developed [1921]. We have developed an approach of TF titration combined with liquid handling and approaches from combinatorial chemistry and fluorescence high throughput screening to help scan and identify blood coagulation interactions [8]. Use of these high throughput data collection and analysis methods allows comparison with simulation. MC simulation provides a tool to understand dynamics and statistics related to a small number of $\mathrm{TF}$ molecules in a small local volume of blood.

\section{Materials and Methods}

\section{Kinetic Monte Carlo}

The Gillespie method [7] allows treatment of reacting systems, from single molecules to high concentrations in a volume of interest. It is governed by the stochastic simulation algorithm, which is based on the probability that a reaction will occur and can be derived from reaction rate equations. The system is governed by two questions: 'What time does the next reaction occur?' and 'What kind of reaction will it be?' The following equations govern the stochastic approach:

$$
\begin{aligned}
& a_{i}(\mu, v) d t=C\left(u_{\mu}, u_{v}\right) X_{\mu} X_{v} d t \\
& C\left(u_{\mu}, u_{\nu}\right) \equiv \frac{k\left(u_{\mu}, u_{\nu}\right)}{V} \\
& \alpha=\sum_{k} a_{k}
\end{aligned}
$$

$$
\begin{aligned}
\tau= & \frac{1}{\alpha} \ln \left(\frac{1}{r_{1}}\right) \\
& \sum_{i=1}^{M-1} a_{i} \leq r_{2} \alpha<\sum_{i=1}^{M} a_{i}
\end{aligned}
$$

Equations 1 and 2 are for two reactants, species $\mu$ and $\nu$, with respective populations $X_{\mu}$ and $X_{\nu}$. Beginning with known populations of all species, equation 1 is the relative probability, $a_{i}$, that any two particles of species $\mu$ and $\nu$ with respective populations $X_{\mu}$ and $X_{\nu}$ will react in the next time interval, $d t$. Equation 2 is the relationship of $C$ to the reaction-rate constant $k$ for any two-reactant reaction $(\mathrm{A}+\mathrm{B} \rightarrow \mathrm{C}$ or $\mathrm{A}+\mathrm{B} \rightarrow \mathrm{C}+\mathrm{D})$ where $V$ is the volume of the system. $V$ is present because the reaction rate constant, $k$, is normally used in differential equations that contain molecular concentrations (molecules per unit volume). $C$ has the units of $\mathrm{s}^{-1}$. If the reaction had three reactant molecules, $V^{2}$ would replace $V$ in equation 2. For one reactant molecule, $C$ equals $k(C=k)$. In equation $3, \alpha$ is the summation of all reaction and aggregation probabilities, $a_{i}$, for all reactions with any number of reactants. $\tau$ in equation 4 is the time step in which the next reaction occurs, where $r_{1}$ is a random number between 0 and 1 . The $M$ determined from equation 5 (where $r_{2}$ is another random number) specifies which reaction occurs.

The MC proceeds as follows: with known populations, calculate equations 1 and 3. Move ahead in time by the time step, $\tau$, determined in equation 4. Determine which reaction or aggregation event occurs via equation 5. Update the populations and begin again. The system of equations in table 1 was programmed into Fortran 90 to run as a stochastic MC. Initial conditions are shown in table 2 for undiluted and 3 -fold diluted whole blood. The equations and constants are identical to those used by Hockin et al. [4]. The default multiplicative linear congruent random number generator used by Fortran was determined to be a poor choice for our simulations because of its relatively short period $\left(=2^{31}-2\right.$, approximately 2 billion), compared to the number of reactions that may take place (sometimes $>10$ billion) and the ability for some seeds to provide even shorter periods. We used the Mersenne Twister (MT) [22] with a long period $\left(=2^{19,937}-1\right.$, approximately $\left.10^{6,000}\right)$ and no known poor seeding. Simulations used to compare against experiments were run with a 3 -fold dilution, while comparison to published deterministic models was run at concentrations without dilution.

There are two ways to determine the number of molecules in a simulation: set the control volume or set a lowest molecule count (LMC). By setting the control volume, one can determine the number of molecules from the initial concentrations (table 2) using Avogadro's number. Since the concentrations are simply a representation of the ratio of the components in any given volume, one can also assign the lowest non-zero initial condition to a set value, or LMC, and back calculate the other initial conditions and the control volume. When using the LMC method to set the volume, the LMC is set to 50 .

Individual simulations (compiled with Absoft Pro FORTRAN 9.0) were distributed to 13 Dell Pentium IV computers using a batch queuing system written in Perl 5.8.4. A 3.3 pl simulation initiated by $25 \mathrm{p} M$ of TF contained $4.83 \times 10^{6}$ total molecules and required $14 \mathrm{~min}$ of CPU time, while a simulation of $1 \mathrm{nl}$ with the same initial concentrations containing $1.46 \times 10^{10}$ molecules required $10 \mathrm{~h}$ of CPU time. 
Table 1. Coagulation cascade

\begin{tabular}{|c|c|c|c|}
\hline Chemical expressions & $\mathrm{k}_{1}$ & $\mathrm{k}_{-1}$ & $\mathrm{k}_{\text {cat }}$ \\
\hline $\mathrm{TF}+\mathrm{VII} \leftrightarrow \mathrm{TF}=\mathrm{VII}$ & $3.2 \times 10^{6} M^{-1} \mathrm{~s}^{-1}$ & $3.1 \times 10^{-3} \mathrm{~s}^{-1}$ & \\
\hline $\mathrm{TF}+\mathrm{VIIa} \leftrightarrow \mathrm{TF}=\mathrm{VIIa}$ & $2.3 \times 10^{7} M^{-1} \mathrm{~s}^{-1}$ & $3.1 \times 10^{-3} \mathrm{~s}^{-1}$ & \\
\hline $\mathrm{TF}=\mathrm{VIIa}+\mathrm{VII} \rightarrow \mathrm{TF}=\mathrm{VIIa}+\mathrm{VIIa}$ & $4.4 \times 10^{5} M^{-1} \mathrm{~s}^{-1}$ & & \\
\hline $\mathrm{Xa}+\mathrm{VII} \rightarrow \mathrm{Xa}+\mathrm{VIIa}$ & $1.3 \times 10^{7} M^{-1} \mathrm{~s}^{-1}$ & & \\
\hline $\mathrm{IIa}+\mathrm{VII} \rightarrow \mathrm{IIa}+\mathrm{VIIa}$ & $2.3 \times 10^{4} M^{-1} \mathrm{~s}^{-1}$ & & \\
\hline $\mathrm{TF}=\mathrm{VIIa}+\mathrm{X} \leftrightarrow \mathrm{TF}=\mathrm{VIIa}=\mathrm{X} \rightarrow \mathrm{TF}=\mathrm{VIIa}=\mathrm{Xa}$ & $2.5 \times 10^{7} M^{-1} \mathrm{~s}^{-1}$ & $1.05 \mathrm{~s}^{-1}$ & $6 \mathrm{~s}^{-1}$ \\
\hline $\mathrm{TF}=\mathrm{VIIa}+\mathrm{Xa} \leftrightarrow \mathrm{TF}=\mathrm{VIIa}=\mathrm{Xa}$ & $2.2 \times 10^{7} M^{-1} \mathrm{~s}^{-1}$ & $19 \mathrm{~s}^{-1}$ & \\
\hline $\mathrm{TF}=\mathrm{VIIa}+\mathrm{IX} \leftrightarrow \mathrm{TF}=\mathrm{VIIa}=\mathrm{IX} \rightarrow \mathrm{TF}=\mathrm{VIIa}+\mathrm{IXa}$ & $1.0 \times 10^{7} M^{-1} \mathrm{~s}^{-1}$ & $2.4 \mathrm{~s}^{-1}$ & $1.8 \mathrm{~s}^{-1}$ \\
\hline $\mathrm{II}+\mathrm{Xa} \rightarrow \mathrm{IIa}+\mathrm{Xa}$ & $7.5 \times 10^{3} M^{-1} \mathrm{~s}^{-1}$ & & \\
\hline IIa + VIII $\rightarrow$ IIa + VIIIa & $2.0 \times 10^{7} M^{-1} \mathrm{~s}^{-1}$ & & \\
\hline VIIIa + IXa $\leftrightarrow$ IXa $=$ VIIIa & $1.0 \times 10^{7} M^{-1} \mathrm{~s}^{-1}$ & $5.0 \times 10^{-3} \mathrm{~s}^{-1}$ & \\
\hline $\mathrm{IXa}=$ VIIIa + X $\leftrightarrow$ IXa $=$ VIIIa $=X \rightarrow$ IXa $=$ VIIIa + Xa & $1.0 \times 10^{8} M^{-1} \mathrm{~s}^{-1}$ & $1.0 \times 10^{-3} \mathrm{~s}^{-1}$ & $8.2 \mathrm{~s}^{-1}$ \\
\hline VIIIa $\leftrightarrow$ VIIIa $_{1} \cdot \mathrm{L}+$ VIIIa $_{2}$ & $6.0 \times 10^{-3} \mathrm{~s}^{-1}$ & $2.2 \times 10^{4} M^{-1} \mathrm{~s}^{-1}$ & \\
\hline $\mathrm{IXa}=\mathrm{VIIIa}=\mathrm{X} \rightarrow \mathrm{VIIIa}_{1} \cdot \mathrm{L}+\mathrm{VIIIa}_{2}+\mathrm{X}+\mathrm{IXa}$ & $1.0 \times 10^{-3} \mathrm{~s}^{-1}$ & & \\
\hline IXa $=$ VIIIa $\rightarrow$ VIIIa $_{1} \cdot \mathrm{L}+$ VIIIa $_{2}+$ IXa & $1.0 \times 10^{-3} \mathrm{~s}^{-1}$ & & \\
\hline $\mathrm{IIa}+\mathrm{V} \rightarrow \mathrm{IIa}+\mathrm{Va}$ & $2.0 \times 10^{7} M^{-1} \mathrm{~s}^{-1}$ & & \\
\hline $\mathrm{Xa}+\mathrm{Va} \leftrightarrow \mathrm{Xa}=\mathrm{Va}$ & $4.0 \times 10^{8} M^{-1} \mathrm{~s}^{-1}$ & $0.2 \mathrm{~s}^{-1}$ & \\
\hline $\mathrm{Xa}=\mathrm{Va}+\mathrm{II} \leftrightarrow \mathrm{Xa}=\mathrm{Va}=\mathrm{II} \rightarrow \mathrm{Xa}=\mathrm{Va}+\mathrm{mIIa}$ & $1.0 \times 10^{8} M^{-1} \mathrm{~s}^{-1}$ & $103 \mathrm{~s}^{-1}$ & $63.5 \mathrm{~s}^{-1}$ \\
\hline $\mathrm{Xa}=\mathrm{Va}+\mathrm{mIIa} \rightarrow \mathrm{Xa}=\mathrm{Va}+\mathrm{IIa}$ & $1.5 \times 10^{7} M^{-1} \mathrm{~s}^{-1}$ & & \\
\hline $\mathrm{Xa}+\mathrm{TFPI} \leftrightarrow \mathrm{Xa}=$ TFPI & $9.0 \times 10^{5} M^{-1} \mathrm{~s}^{-1}$ & $3.6 \times 10^{-4} \mathrm{~s}^{-1}$ & \\
\hline $\mathrm{TF}=\mathrm{VIIa}=\mathrm{Xa}+\mathrm{TFPI} \leftrightarrow \mathrm{TF}=\mathrm{VIIa}=\mathrm{Xa}=\mathrm{TFPI}$ & $3.2 \times 10^{8} M^{-1} \mathrm{~s}^{-1}$ & $1.1 \times 10^{-4} \mathrm{~s}^{-1}$ & \\
\hline $\mathrm{TF}=\mathrm{VIIa}+\mathrm{Xa}=\mathrm{TFPI} \rightarrow \mathrm{TF}=\mathrm{VIIa}=\mathrm{Xa}=\mathrm{TFPI}$ & $5.0 \times 10^{7} M^{-1} \mathrm{~s}^{-1}$ & & \\
\hline $\mathrm{Xa}+\mathrm{ATIII} \rightarrow \mathrm{Xa}=$ ATIII & $1.5 \times 10^{3} M^{-1} \mathrm{~s}^{-1}$ & & \\
\hline $\mathrm{mIIa}+$ ATIII $\rightarrow$ mIIa $=$ ATIII & $7.1 \times 10^{3} M^{-1} \mathrm{~s}^{-1}$ & & \\
\hline $\mathrm{IXa}+\mathrm{ATIII} \rightarrow \mathrm{IXa}=$ ATIII & $4.9 \times 10^{2} M^{-1} \mathrm{~s}^{-1}$ & & \\
\hline $\mathrm{IIa}+\mathrm{ATIII} \rightarrow$ IIa $=$ ATIII & $7.1 \times 10^{3} M^{-1} \mathrm{~s}^{-1}$ & & \\
\hline $\mathrm{TF}=\mathrm{VIIa}+\mathrm{ATIII} \rightarrow \mathrm{TF}=\mathrm{VIIa}=\mathrm{ATIII}$ & $2.3 \times 10^{2} M^{-1} \mathrm{~s}^{-1}$ & & \\
\hline
\end{tabular}

This list of reactions contains chemical reactions and related kinetics for the stochastic Monte Carlo simulation. The on-rate $\left(\mathrm{k}_{1}\right)$, off-rate $\left(\mathrm{k}_{-1}\right)$, and catalytic reaction rate $\left(\mathrm{k}_{\mathrm{cat}}\right)$ are from Hockin et al. [4], with their respective reactions.

\section{Blood Coagulation Monitoring}

Sodium citrate solution (Sigma-Aldrich, St. Louis, Mo., USA), thrombin (Enzyme Research Labs, South Bend, Ind., USA), corn trypsin inhibitor (CTI, Haematologic Technologies, Essex Junction, Vt., USA), recombinant lipidated human TF (American Diagnostica, Stamford, Conn., USA), calcium chloride (Fisher Scientific, Pittsburgh, Pa., USA), convulxin (Centerchem, Norwalk, Conn., USA), were stored following the manufacturers' recommendations. The fluorogenic substrate for thrombin, boc-Val-Pro-Arg-methylcoumarin-7-amide (boc-VPR-MCA), was obtained from Bachem Bioscience (King of Prussia, Pa., USA). Unless otherwise noted, all dilutions were made using Hank's balanced salt solution (HBSS, 1X, without calcium chloride, magnesium chloride, magnesium sulfate, or phenol red; Invitrogen Corp., Carlsbad, Calif., USA). Human blood was collected from healthy adult donors and anticoagulated with sodium citrate ( 9 parts blood to 1 part sodium citrate). Corn trypsin inhibitor was added at a final concentration of $50 \mu \mathrm{g} / \mathrm{ml}$ to block the intrinsic pathway by blocking factor XIIa [23].

\section{High Throughput 384-Well Coagulation Assay}

Reactions were combined in Costar 384-well plates (Fisher Scientific) using a Perkin-Elmer (Boston, Mass., USA) Multiprobe II liquid handling system with 4 variable spacing pipetting heads. Each reaction well of the 384-well plate contained a final reaction volume of $60 \mu \mathrm{l}$. To begin, $10 \mu \mathrm{l}$ of lipidated TF was added to each well. Next, a volume of $10 \mu \mathrm{l}$ of HBSS was added to the wells. Then, $20 \mu \mathrm{l}$ of CTI-treated, citrated whole blood was delivered into all wells, followed immediately by addition of $20 \mu \mathrm{l}$ of $30 \mathrm{mM}$ calcium chloride and $30 \mu M$ boc-VPR-MCA solution with or without $30 \mathrm{n} M$ convulxin to recalcify and initiate each reaction (final concentrations: $10 \mathrm{~m} M \mathrm{CaCl}_{2}, 10 \mu M$ boc-VPR-MCA, $16.7 \mu \mathrm{g} / \mathrm{ml}$ of CTI, 0 or $10 \mathrm{n} M$ of convulxin in each $60 \mu \mathrm{l}$ of 3-fold diluted citrated whole blood). Fluorescence (EX: $390 \mathrm{~nm}$; EM: $460 \mathrm{~nm}$ ) from cleaved 7-amino-methylcoumarin was detected using a ThermoLabsystems Ascent Fluoroskan FL (Franklin, Mass, USA) plate reader. Each single reaction condition was repeated in replicates on the same plate 12 times using a single donor's blood. In experimental wells, to prevent spatial differences between wells, the reactions and their repeats were placed in a spiral conformation from the 
Table 2. Initial concentrations. Initial conditions for all components in the stochastic Monte Carlo simulations for undiluted whole blood and whole blood diluted 3-fold

\begin{tabular}{|c|c|c|}
\hline Species & $\begin{array}{l}\text { Initial } \\
\text { concentration, } M\end{array}$ & $\begin{array}{l}3 \text {-fold diluted initial } \\
\text { concentration, } M\end{array}$ \\
\hline $\mathrm{TF}$ & Varied & Varied \\
\hline VII & $1.00 \mathrm{E}-08$ & 3.33E-09 \\
\hline $\mathrm{TF}=\mathrm{VII}$ & 0.0 & 0.0 \\
\hline VIIa & $1.00 \mathrm{E}-10$ & $3.33 \mathrm{E}-11$ \\
\hline $\mathrm{TF}=\mathrm{VIIa}$ & 0.0 & 0.0 \\
\hline $\mathrm{Xa}$ & 0.0 & 0.0 \\
\hline IIa & 0.0 & 0.0 \\
\hline $\mathrm{X}$ & $1.60 \mathrm{E}-07$ & $5.33 \mathrm{E}-08$ \\
\hline $\mathrm{TF}=\mathrm{VIIa}=\mathrm{X}$ & 0.0 & 0.0 \\
\hline $\mathrm{TF}=\mathrm{VIIa}=\mathrm{Xa}$ & 0.0 & 0.0 \\
\hline IX & $9.00 \mathrm{E}-08$ & $3.00 \mathrm{E}-08$ \\
\hline $\mathrm{TF}=\mathrm{VIIa}=\mathrm{IX}$ & 0.0 & 0.0 \\
\hline IXa & 0.0 & 0.0 \\
\hline II & $1.40 \mathrm{E}-06$ & 4.67E-07 \\
\hline VIII & $7.00 \mathrm{E}-10$ & $2.33 \mathrm{E}-10$ \\
\hline VIIIa & 0.0 & 0.0 \\
\hline $\mathrm{IXa}=\mathrm{VIIIa}$ & 0.0 & 0.0 \\
\hline $\mathrm{IXa}=\mathrm{VIIIa}=\mathrm{X}$ & 0.0 & 0.0 \\
\hline VIIIa1-L & 0.0 & 0.0 \\
\hline VIIIa2 & 0.0 & 0.0 \\
\hline $\mathrm{V}$ & $2.00 \mathrm{E}-08$ & 6.67E-09 \\
\hline $\mathrm{Va}$ & 0.0 & 0.0 \\
\hline $\mathrm{Xa}=\mathrm{Va}$ & 0.0 & 0.0 \\
\hline $\mathrm{Xa}=\mathrm{Va}=\mathrm{II}$ & 0.0 & 0.0 \\
\hline mIIa & 0.0 & 0.0 \\
\hline TFPI & $2.50 \mathrm{E}-09$ & 8.33E-10 \\
\hline $\mathrm{Xa}=\mathrm{TFPI}$ & 0.0 & 0.0 \\
\hline $\mathrm{TF}=\mathrm{VIIa}=\mathrm{Xa}=\mathrm{TFPI}$ & 0.0 & 0.0 \\
\hline ATIII & $3.40 \mathrm{E}-06$ & $1.13 \mathrm{E}-06$ \\
\hline $\mathrm{Xa}=\mathrm{ATIII}$ & 0.0 & 0.0 \\
\hline mIIa = ATIII & 0.0 & 0.0 \\
\hline $\mathrm{IXa}=\mathrm{ATIII}$ & 0.0 & 0.0 \\
\hline IIa = ATIII & 0.0 & 0.0 \\
\hline $\mathrm{TF}=\mathrm{VIIa}=\mathrm{ATIII}$ & 0.0 & 0.0 \\
\hline
\end{tabular}

center of the plate. A volume of $1 \mathrm{ml}$ of citrated whole blood allowed 50 reactions with a $10-\mathrm{ml}$ blood draw, amply sufficient for 384 reactions on a single plate.

\section{Thrombin Detection and Data Analysis}

With full conversion of boc-VPR-MCA after the propagation phase, each well reached maximal fluorescence $\left(F_{\max }\right)$. Using each well's lowest fluorescence $\left(\mathrm{F}_{0}\right)$, the data was normalized between 0 and 1 using $F^{\prime}(t)=\left(F(t)-F_{0}\right) /\left(F_{\max }-F_{0}\right)$ to minimize well-to-well liquid handling and detection variation. Analysis of the normalized thrombin substrate conversion traces included extraction of the initiation time $\left(\mathrm{T}_{\mathrm{i}}\right)$, defined as the time when $\mathrm{F}\left(\mathrm{T}_{\mathrm{i}}\right)=1.03\left(\mathrm{~F}_{0}\right)$ or $\mathrm{F}^{\prime}$ $\left(\mathrm{T}_{\mathrm{i}}\right)=0.03$, the time of maximum rate of thrombin production $\left(\mathrm{T}_{\max }\right)$, and the maximum rate of thrombin production $\left(\mathrm{dF}^{\prime} / \mathrm{dt}_{\max }\right)$.
The instantaneous rate of thrombin production for each well was determined by fitting a curve to the normalized data, and determining the derivative at each time. $\mathrm{T}_{\max }$ and $\mathrm{dF}^{\prime} / \mathrm{dt}_{\max }$ are determined when the derivative $\left(\mathrm{dF}^{\prime} / \mathrm{dt}\right)$ is maximal and does not necessarily correspond to the time when substrate is $50 \%$ consumed. The substrate hydrolysis rate is linearly dependent on thrombin concentration only at low levels of conversion. $\mathrm{T}_{\max }$ and $\mathrm{dF}^{\prime} / \mathrm{dt}_{\max }$ are data analysis tools to define when thrombin production becomes explosive. In calibration studies, $\mathrm{dF}^{\prime} / \mathrm{dt}_{\max }$ was determined to occur at approximately $1.49 \mathrm{U} / \mathrm{ml}$ of thrombin for convulxin-added reactions.

\section{Results}

\section{Stochastic Results}

The stochastic effects on averages and standard deviations for MC simulations of undiluted whole blood triggered with $5 \mathrm{p} M$ TF reveal the effects of simulation volume (fig. 1). Total thrombin is defined as thrombin (IIa) and meizothrombin (mIIa) with meizothrombin multiplied by a factor of 1.2 because of meizothrombin's $20 \%$ greater activity toward substrates [4]. Figure 1a shows the average for 22 simulations at $1 \mathrm{nl}$ simulation volume over $1,200 \mathrm{~s}$. Figure $1 \mathrm{~b}$ shows each individual simulation of $5 \mathrm{p} M$ TF. The stochastic effects on these simulations are evident in the average peak height and the standard deviation of the time of maximum. The average peak height (fig. 1a) is slightly lower than the peak of each simulation (fig. 1b) due to averaging effects. The average peak height occurs at about $350 \mathrm{n} M$, but the peaks of each simulation run all reach about $390 \mathrm{n} M$ thrombin. Stochastic variation and random events lead to differences in the onset of initiation time, which lead to the averages of stochastic simulations being low. Stochastic variations and random events can become even more prevalent in smaller simulation volumes. Figures $1 \mathrm{c}$ and $1 \mathrm{~d}$ show equivalent data $(\mathrm{n}=35)$ in a $16.6 \mathrm{pl}$ simulation volume. This volume would contain approximately 5 platelets $(300,000$ platelets/ $\mu$ l). The average (fig. 1c) is not representative of each individual simulation (fig. 1d) because of the large stochastic variability in the initiation time caused by the larger prevalence of stochastic variation at this small volume of initiating levels of factor Xa and thrombin. This variation leads to an average with a very broad peak even though each simulation contains a single distinct peak in thrombin concentration. Over hundreds or thousands of $16.6 \mathrm{pl}$ simulations, the average behavior for these wellmixed, isotropic simulations, would begin to approach that seen in figure 1a. 


\section{Model Validation}

Stochastic MC simulations were compared (fig. 2) with published deterministic solutions of the Mann model [4]. Deterministic solutions of the Mann model (fig. 2a, c) agreed with the 1-nl volume MC solutions (fig. 2b, d). Although each MC simulation had the same initial conditions, the results varied due to probabilities and random events. All the MC plots were very close to their deterministic counterparts except for 5-p $M$ TF. The thrombin peak for the 5-p $M$ TF curves averaged about $350 \mathrm{n} M$, while the deterministic peak approached $390 \mathrm{n} M$. As explained previously, this was due to averaging stochastic differences in the onset of thrombin production. Stochastic variations existed at low concentrations for intrinsic factor Xase complex (IXa-VIIIa) because the simulation approached 1 or 2 molecules within the simulated volume $(1 \mathrm{nl}, 1$ molecule in $1 \mathrm{nl} \approx 1.7 \times$ $10^{-15} \mathrm{M}$ ). Factor Xa concentrations were low because the stochastic variations in the intrinsic factor Xase complex delay the onset of factor Xa production. Other comparisons between the deterministic and MC simulations were made (see fig. 5 to 9 of Hockin et al. [4]) and matched well.

\section{Clot Initiation}

Initiation time was set as the time for which the simulation reaches $0.05 \mathrm{U} / \mathrm{ml}(0.45 \mathrm{n} M)$ thrombin. This concentration of thrombin causes $\sim 50 \%$ release of platelet granules and $\sim 50 \%$ display of P-selectin [24, 25]. Figure 3 shows individual thrombin simulation data for simulations initiated with $0.186 \mathrm{p} M$ TF in 3-fold diluted whole blood. At this critical activating concentration of TF, 50\% of the simulations initiated within $1 \mathrm{~h}\left(\mathrm{~T}_{\mathrm{i}}<1 \mathrm{~h}\right)$. Simulations that initiated are represented in gray, while black lines represent simulations that did not initiate. The dashed line represents $0.05 \mathrm{U} / \mathrm{ml}$ thrombin, the initiation cut-off. The inset plot shows the log of thrombin concentration plotted versus time.

Figure 4a plots the initiation times for MC simulations that initiate $\left(\mathrm{T}_{\mathrm{i}}<1 \mathrm{~h}\right)$. Table 3 contains the TF concentrations plotted and their respective probabilities of initiation $(0.05 \mathrm{U} / \mathrm{ml})$ and reaching the maximum rate of fluorescence generation $(1.49 \mathrm{U} / \mathrm{ml})$ within $1 \mathrm{~h}\left(\mathrm{~T}_{\max }<\right.$ $1 \mathrm{~h})$. Figure $4 \mathrm{~b}$ plots the probability of initiation versus TF concentration. The simulation predicts a $100 \%$ probability of initiation at $0.7 \mathrm{p} M \mathrm{TF}, 50 \%$ probability of initiation at $0.186 \mathrm{p} M \mathrm{TF}$ and $0 \%$ probability of initiation at $0.07 \mathrm{p} M \mathrm{TF}$.

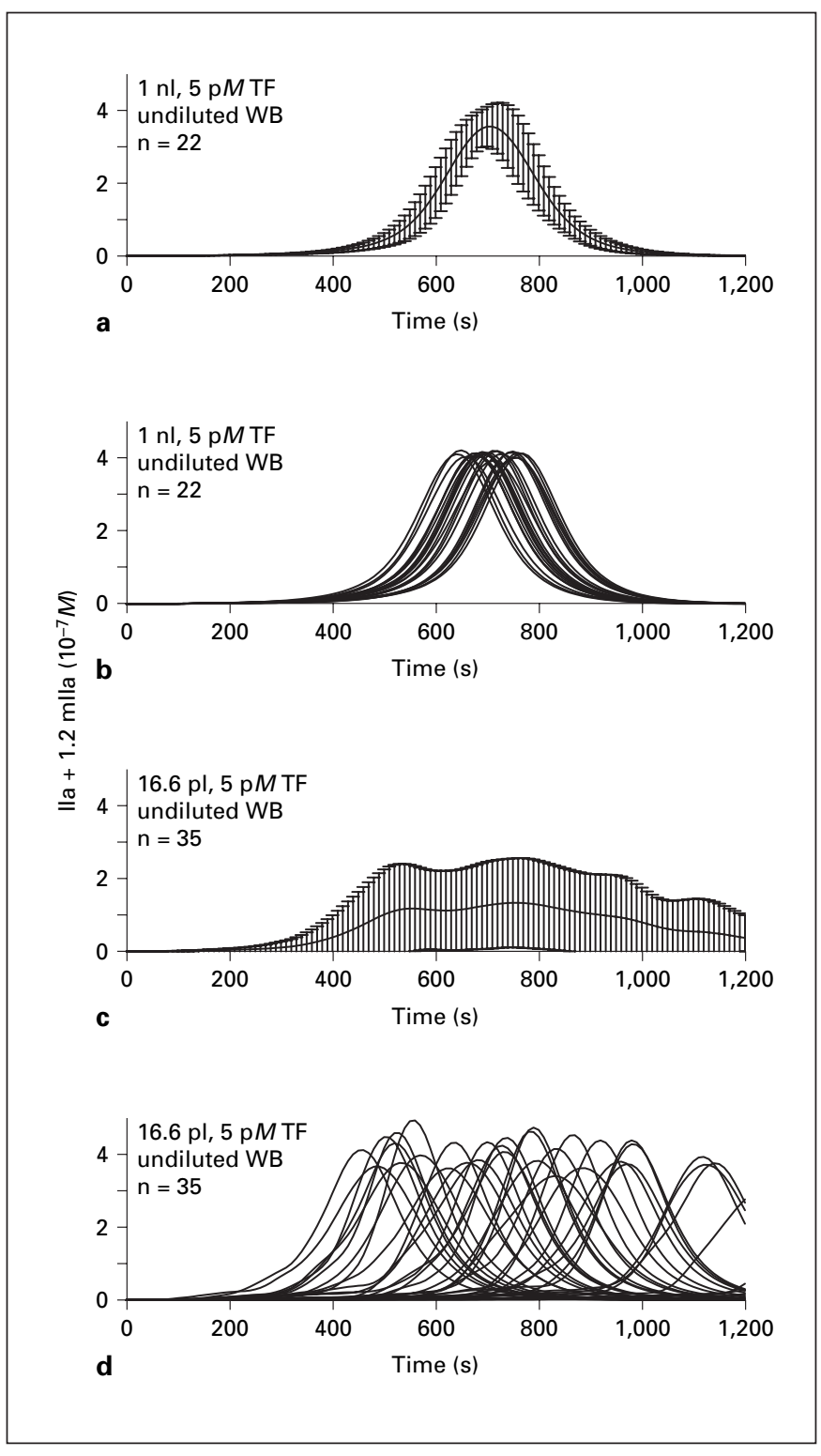

Fig. 1. Total thrombin initiated by $5-\mathrm{p} M$ TF simulations. For $\mathrm{n}=$ 22, $1 \mathrm{nl}$ simulations of undiluted whole blood coagulation activated by $5 \mathrm{p} M \mathrm{TF}$, $\mathbf{a}$ is the mean of total thrombin generation, while b displays each simulation. The mean of the simulations displays a lower maximum peak than the simulations themselves. The time shift of the initiation time is caused by stochastic variations, which leads to the lower average peak. Identical simulations in $\mathbf{c}$ and $\mathbf{d}$ are simulated at a smaller control volume, $16.6 \mathrm{pl}$ with $\mathrm{n}=35$. With approximately $1.7 \%$ of the volume of the previous simulations, $\mathbf{c}$ does not show a single well-defined peak. Also it contains significantly larger standard deviations because of significantly higher variation in the initiation time for the simulation because fewer molecules are present in the smaller volume. Note that only 32 of the 35 simulations represented in $\mathbf{d}$ reach their maximum value within the $1,200 \mathrm{~s}$ shown. 


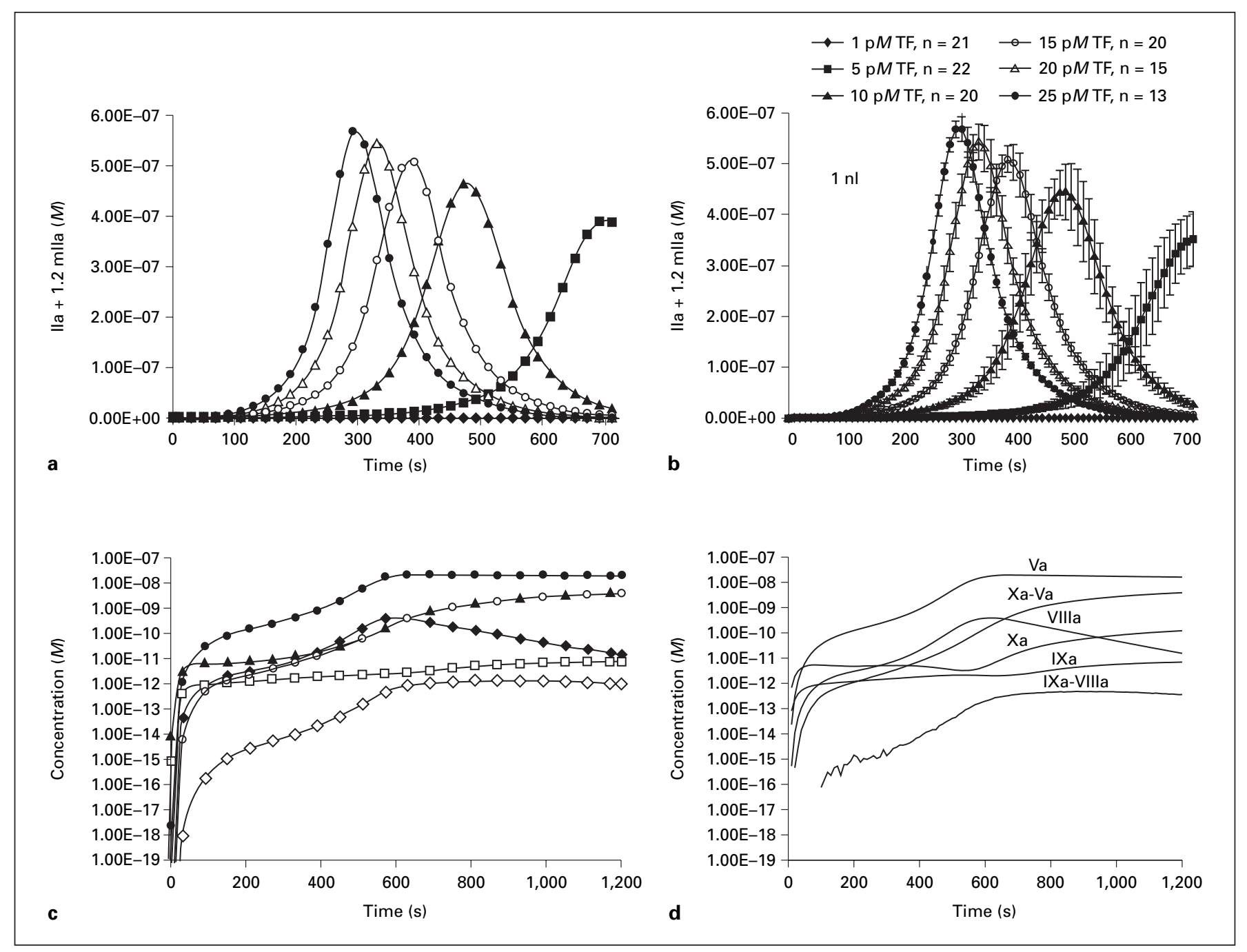

Fig. 2. Comparison of coagulation results. a, c Deterministic solutions of the Hockin coagulation model [4]. b, d Comparable results generated by stochastic Monte Carlo (MC) simulation. All MC simulations shown here were $1 \mathrm{nl}$ of simulated, undiluted whole blood coagulation. These stochastic simulation results are often slightly lower than Hockin's results because of the nature of the variation in the simulations. $\mathbf{a}$ and $\mathbf{b}$ plot total thrombin (IIa $+1.2 \mathrm{mIIa}$ ) as a function of time for varying TF concentrations. The factor of 1.2 for the mIIa is included for meizothrombin's increased activity towards substrates [4]. Each simulation was run with initial concentrations shown in table 2 with the TF concentration of $1 \mathrm{p} M$ (filled diamonds), $5 \mathrm{p} M$ (filled squares), $10 \mathrm{p} M$ (filled triangles), $15 \mathrm{p} M$ (open circles), $20 \mathrm{p} M$ (open triangles), and $25 \mathrm{p} M$ (filled circles). $\mathbf{c}$ and $\mathbf{d}$ provide a logarithmic view of the metabolite concentrations during coagulation activated by $5 \mathrm{p} M \mathrm{TF}$ : Xa (filled triangles), Va (filled circles), intrinsic Xase (open diamonds), prothrombinase (open circles), IXa (open squares), and VIIIa (filled diamonds). In the $\mathrm{MC}$ simulation (d), intrinsic Xase exhibits stochastic variations caused by one or two molecules being generated between time steps. As the MC requires a set volume $(1 \mathrm{nl})$, stochastic variations are visible at these levels ( 1 molecule in $\left.1 \mathrm{nl} \approx 1.7 \times 10^{-15} M\right)$. This stochastic variation causes visible differences in factor Xa generation.

\section{TF Titration and Simulation Comparison}

Simulations and experiments were run at a titration of TF concentrations and compared. At each TF concentration, experiments were run with and without convulxin. Convulxin is a potent GPVI activator [26]. The Mann model [4] assumes full platelet activation at $\mathrm{t}=0$, and this state can be established experimentally by using convulxin to stimulate platelet activation. In 3 donors and 936 individual reactions, the average trends in $\mathrm{T}_{\mathrm{i}}($ fig. 5a) and $\mathrm{T}_{\max }$ (fig. $5 \mathrm{~b}$ ) with $\mathrm{TF}$ titration ( $\pm 10 \mathrm{n} M$ convulxin) were 
Fig. 3. $50 \%$ of simulations initiate at $0.186 \mathrm{p} M \mathrm{TF}$. In 3-fold diluted whole blood, $0.186 \mathrm{p} M$ TF causes $50 \%$ of the simulations to initiate within $1 \mathrm{~h}$. The initiation concentration of $0.05 \mathrm{U} / \mathrm{ml}$ IIa (dashed line) was chosen [24, 25] for its relation to the IIa concentration required for platelet activation through PAR1 and PAR4 [29]. Simulations that initiate within $1 \mathrm{~h}$ are shown in gray. Black lines are simulations that do not initiate. The inset figure illustrates the data on a logarithmic scale. On this scale, the marginal increase in thrombin concentration before initiation is visible, as is the change to significantly steeper slopes as initiation progresses.

Fig. 4. Initiation probability. Initiation time for low-volume simulations at a 3-fold dilution of whole blood are shown in (a). All simulations were run using a lowest molecule count (LMC) of 50 molecules of TF, which varies the simulation volume between $6 \mathrm{pl}$ for the highest TF concentration to $4 \mathrm{nl}$ at the lowest TF concentration $(0 \mathrm{pM}$ TF simulations were run at $2.5 \mathrm{pl}$ with the LMC being assigned to VIIa). Not all simulations initiated below $0.692 \mathrm{p} M$ TF. a Indicates the initiation time for the simulations that initiate within $1 \mathrm{~h}$ for various TF concentrations within a log scale of concentrations. $\mathbf{b}$ Indicates the fraction of simulations that initiated (table 3 ) with the point that $50 \%$ of simulations initiated occurring near $0.186 \mathrm{p} M \mathrm{TF}$.
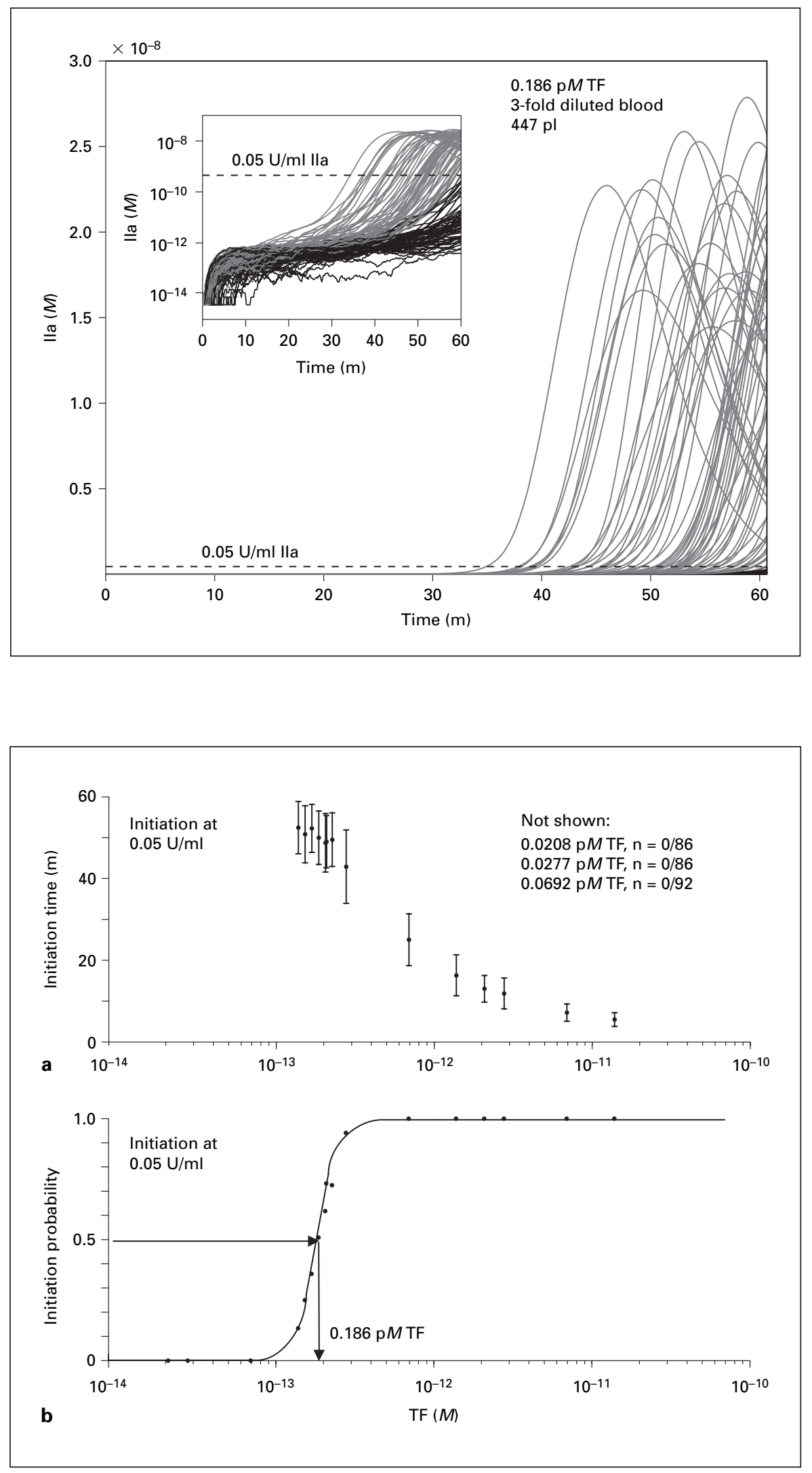
Table 3. Initiation and maximum rate probability

\begin{tabular}{llll}
\hline $\begin{array}{l}\text { TF concentra- } \\
\text { tion }(\mathrm{p} M)\end{array}$ & $\begin{array}{l}\text { Total } \\
\text { number }\end{array}$ & $\begin{array}{l}\text { Initiation } \\
\text { probability }\end{array}$ & $\begin{array}{l}\text { Max rate } \\
\text { probability }\end{array}$ \\
\hline 0 & 76 & 0.000 & 0.000 \\
0.0208 & 86 & 0.000 & 0.000 \\
0.0277 & 86 & 0.000 & 0.000 \\
0.0692 & 92 & 0.000 & 0.000 \\
0.138 & 90 & 0.133 & 0.033 \\
0.1524 & 100 & 0.250 & 0.100 \\
0.1682 & 120 & 0.358 & 0.150 \\
0.1857 & 120 & 0.508 & 0.325 \\
0.2051 & 89 & 0.618 & 0.371 \\
0.208 & 82 & 0.732 & 0.537 \\
0.2264 & 40 & 0.725 & 0.450 \\
0.277 & 68 & 0.941 & 0.838 \\
0.692 & 84 & 1.000 & 1.000 \\
1.38 & 56 & 1.000 & 1.000 \\
2.08 & 54 & 1.000 & 1.000 \\
2.77 & 62 & 1.000 & 1.000 \\
6.92 & 60 & 1.000 & 1.000 \\
13.84 & 102 & 1.000 & 1.000 \\
\hline
\end{tabular}

For various initiating tissue factor concentrations in 3-fold dilute blood coagulation simulation, the total number of simulations run at each concentration are shown as well as the fraction (initiation probability) of simulations that reach the initiating thrombin concentration $(0.05 \mathrm{U} / \mathrm{ml})$ and the fraction (max rate probability) of simulations that reach $1.4911 \mathrm{U} / \mathrm{ml}$ thrombin, the concentration of thrombin where the maximum rate of fluorescence conversion occurs in related experiments. $50 \%$ probability of initiation was deduced to occur near $0.1857 \mathrm{p} M$ of tissue factor.

compared against simulation in clotting time analysis when using 3-fold diluted whole blood. Each experimental data bar with or without convulxin contains 36 experiments per TF concentration averaged over 3 donors ( $\mathrm{n}=12 /$ donor/TF concentration). In thrombin time testing with whole blood, it is commonly assumed that platelet activation occurs promptly after addition of high TF doses. Platelet activation by convulxin decreased $T_{i}$ and $\mathrm{T}_{\max }$ over the entire range of the TF titration. In fig. 5a, the experimental effects of TF can be detected at $0.28 \mathrm{pM}$ $(p<0.05)$ in the absence of convulxin. With coaddition of convulxin, the effects of TF activation can be clearly detected at $0.07 \mathrm{p} M$ (or approximately 1.6 platelets per molecule of TF), demonstrating the sensitivity of the experimental approach as well as the enormous amplifying action of protease cascades in activated blood. All simulations with TF concentrations $>1.38 \mathrm{p} M$ TF match very well with convulxin-added experiments, as expected. The simulation, however, begins to fail at subpicomolar
$(<1 \mathrm{p} M)$ TF concentrations, and the reactions did not initiate within $1 \mathrm{~h}$ at extremely low $(<0.1 \mathrm{p} M)$ TF concentrations. The MC showed excellent agreement to experiment at $\geq 1.38 \mathrm{p} M \mathrm{TF}$ with convulxin. However, at lower concentrations $<1 \mathrm{p} M \mathrm{TF}$, the simulation and experiment did not agree. Figure $5 \mathrm{~b}$ compares the time of maximum rate as experimentally measured versus the time the simulation reaches $1.49 \mathrm{U} / \mathrm{ml}(13.4 \mathrm{n} M)$ thrombin. As previously mentioned, the simulation assumes maximal platelet activation [4], so from calibration studies, the time of maximum rate, as measured experimentally by fluorescence in convulxin-added experiments, occurs at approximately $1.49 \mathrm{U} / \mathrm{ml}$ thrombin. As with initiation time (fig. 5a), convulxin provides similar enhancement. The simulation and experiment do not agree at low $\mathrm{TF}<1 \mathrm{p} M \mathrm{TF}$.

\section{Discussion}

We have converted a deterministic model developed by Hockin et al. [4] into a stochastic MC simulation and compared it with experiments. Stochastic MC simulations reveal small stochastic variations in thrombin initiation time (fig. 1). These variations occur when several reactions have similar probabilities of occurring. Depending on which reaction occurs, this can cause thrombin generation at the current time step or a delay in thrombin generation to a future time step. These stochastic variances cause averages of the simulation data to appear lower. As expected, small-volume simulations also amplify stochastic variations. A clump of 5 platelets in a resident volume on the order of $10 \mathrm{pl}$ will display highly stochastic clotting, even at $5 \mathrm{p} M$ TF (fig. 1d). Figures $1 \mathrm{a}$ and $1 \mathrm{~b}$ are examples of what occurs macroscopically, within the relative large volumes of blood ( $\geq 1 \mathrm{nl}$ ), lacking prominent stochastic variability. Figure 1d is an example of what occurs microscopically within much smaller, isolated subvolumes of blood where stochastic effects at low molecular populations are present. For instance, if one was to extend the stochastic MC into a 2D or 3D simulation with spatial gradients, figure $1 \mathrm{~d}$ would represent what is occurring locally in many different control volumes of $16.6 \mathrm{pl}$ within a 2D or 3D space of a larger control volume. The impact of local 'hotspots' remains to be studied.

Despite these variances, the stochastic MC compares well with the original deterministic model. When the MC is compared with Hockin's [4] deterministic model(fig. 2), two differences caused by stochastic simulation are apparent. In figure $2 \mathrm{~b}$, the $5 \mathrm{p} M$ TF peak is low. Again, this 


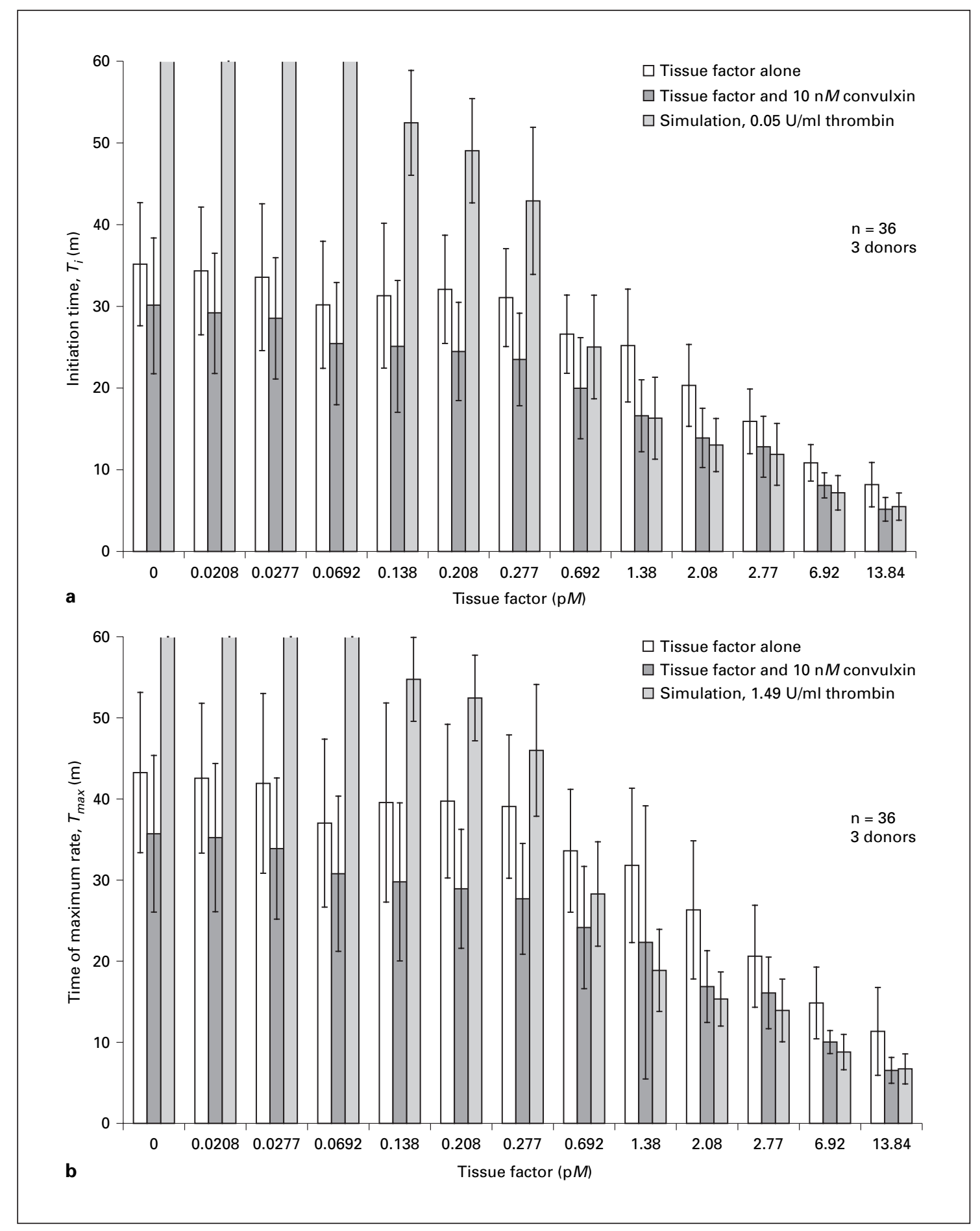

Fig. 5. Comparison of simulation and experiment. Initiation time and time of maximum rate were compared between experiments and simulations. Experimental results represent thrombin generation, as indicated by boc-VPR-MCA cleavage as a function of TF addition to CTI-treated, 3-fold diluted whole blood with or without convulxin for $\mathrm{n}=36$ using 3 donors. For simulation results, initial conditions were diluted 3 -fold to mimic the conditions present during the experimentation. All simulations were run using a lowest molecule count (LMC) of 50 molecules of TF, which varies the simulation volume between $6 \mathrm{pl}$ for the highest TF concentration to $4 \mathrm{nl}$ at the lowest TF concentration ( 0 - $\mathrm{p} M$ TF simulations were run at $2.5 \mathrm{pl}$ with the LMC being assigned to VIIa). The simulation initiation time is defined as the time that the thrombin concentration surpasses $0.05 \mathrm{U} / \mathrm{ml}[24,25]$. a Experiments and model coincide well for convulxin experiments for picomolar levels of TF. At $1.38 \mathrm{p} M$ TF, $\mathrm{p}=0.76$ for Student's $\mathrm{t}$ test between simulation and 
was caused by averaging data. Intrinsic Xase complex has a very jagged profile at short times $(<400 \mathrm{~s}$, fig. $2 \mathrm{~d})$. This occurs because the simulation is modeling small numbers of intrinsic Xase complex at these short times due to volume limitations. Stochastic simulation deals with the molecules in a set volume. For the case in figure $2 \mathrm{~d}$, this volume is $1 \mathrm{nl}$. Partial molecules do not exist in a stochastic simulation, so only integer values of molecules are allowed. Therefore, the smallest 'measureable' amount of any species in figure $2 \mathrm{~d}$ is 1 molecule in $1 \mathrm{nl}$, or approximately $1.7 \times 10^{-15} \mathrm{M}$. So in the femtomolar $\left(10^{-15} M\right)$ range of figure $2 \mathrm{~d}$, the jagged profile is caused by the existence of 1,2 , or 3 molecules during time steps.

Not all simulations initiate for a criteria of $0.05 \mathrm{U} / \mathrm{ml}$ thrombin within $1 \mathrm{~h}$. Simulation initiation was determined to be the time when thrombin generation reached $0.05 \mathrm{U} / \mathrm{ml}(0.45 \mathrm{nM})[24,25]$. Many simulations can be run to determine initiation probabilities (table 3 ). $0.186 \mathrm{p} M$ TF predicts $50 \%$ probability of initiation of coagulation (fig. 3) with $100 \%$ probability of initiation at $0.7 \mathrm{p} M \mathrm{TF}$ and $0 \%$ probability of initiation at $0.07 \mathrm{p} M$ TF (fig. 4b). However, as can be seen from figure 5, the simulation does not accurately predict experimental coagulation initiation at subpicomolar levels of TF. New reactions and interactions are needed in order to simulate blood clotting at $\mathrm{TF}<1 \mathrm{p} M$.

Figure 5 compares the stochastic simulation to experimental data. The simulation matches very well with convulxin-added experimental sample at picomolar levels of added TF. However, at lower levels of TF, the model breaks down. At femtomolar levels $\left(<70 \times 10^{-15} \mathrm{M}\right)$ of $\mathrm{TF}$, the model fails to initiate, and values below $0.692 \mathrm{p} M$ TF may be skewed lower than the actual average initiation time because not all of the simulations reached the initiation concentration $(0.05 \mathrm{U} / \mathrm{ml})$ within the simulated hour. At these low levels of TF, corn trypsin inhibitortreated (factor XIIa inhibitor) blood coagulates in the ab-

experiment; however, at $0.692 \mathrm{p} M \mathrm{TF}, \mathrm{p}<0.0005$, therefore as TF concentrations decrease, the simulation does not accurately predict experimental results. In $\mathbf{b}$, the time of maximum rate for the simulation is defined as the time for the maximum rate of fluorescence production at $1.49 \mathrm{U} / \mathrm{ml}$ (from calibration studies, data not shown). Similar to initiation time (a), the model is predictive for blood clotting activated by convulxin with picomolar levels of TF (at $1.38 \mathrm{p} M$ TF, $\mathrm{p}=0.30$ ), but for lower TF concentrations, the simulation is inaccurate (at $0.692 \mathrm{p} M \mathrm{TF}, \mathrm{p}<0.005$ ).

Coagulation Modeling and Experiment sence of added TF, but with highly variable kinetics under replicate experimental conditions. Although it seems that the stochastic simulation could replicate these highly variable kinetics as evidenced by figure 1 , it does not have the capability to self-clot, as evidenced by standing blood. Without TF, the initial formation of Xa may be from trace levels of IXa, Xa, or IIa in drawn blood, neutrophil protease release to activate $\mathrm{X}$ a via cathepsin $\mathrm{G}$ or elastase, vessel-wall-derived TF from phlebotomy, blood-borne TF [27], or VIIa-cofactor activity [28], monocyte expression of TF during the incubation, or some combination of the above. Combining the model with experimentation may lead to discovering what mechanisms or initial conditions are missing from the model that give rise to this spontaneous coagulation.

Also, the experimental technique demonstrated that the state of platelet activation at $t=0$ effects the initiation phase $\left(T_{i}\right)$ and propagation phase $\left(T_{\max }\right)$ of coagulation. Activation of platelets with convulxin resulted in initiation times similar to that achieved by adding $\sim 0.28 \mathrm{pM}$ TF without convulxin. If platelet activation with convulxin were to result in the exposure of an activity (cofactor or enzyme) and that activity were to be TF or TF-like in function, then the effect of that activity appears to be equivalent to subpicomolar levels of TF. The simulation was more predictive of the convulxin-added data, as expected. Kinetics simulated assumed maximal platelet activation. Updates to the simulation should include reactions to account for platelet function during clotting. The experimental method provides a tool to explore reaction mechanisms, where a given molecule, protein, receptor, or cell (e.g., thrombin, GPIb, PARs, platelets) may have multiple modulatory roles in clotting, depending on the level of input TF. The use of combinatorial reaction analysis provides for highly constrained data sets, where certain reactions are greatly activated or inhibited, while other variables are also adjusted. Such data sets become useful for updating mathematical models such as the one presented here.

Expanding the model into a 2D or 3D model with or without convective flow and adding a vessel wall is extremely desirable but challenging. Spatial coagulation could be studied in a flowing system with surface deposition. Also for future 2D and 3D modeling with convective-diffusive transport, the volume resolution is dependent, in part, on prevailing TF levels.

In figure $2 \mathrm{~d}$, the observation was made previously that at low volumes, variance in the profile is caused by the appearance of one or two molecules. Figure 1 shows that volume can play a major role in the stochastic nature of 
reactions. Assay miniaturization is of particular concern in the face of this. Many microarrays are developed in control volumes less than $2 \mathrm{nl}$. Is there a limit to how small microarrays can be made, and can stochastic modeling predict changes to assay kinetics at these low volumes? Figure 5 shows experimental data from blood coagulation high throughput assays developed in $60-\mu 1$ reactions developed in a 384-well plate. Clearly, $\mathrm{pl}$ and $\mathrm{nl}$ volumes will display stochastic variation.
The objective of this research was to establish a quantitative understanding of blood stability in both experiment and simulation. We have developed a technique to quickly scan and profile coagulation interactions experimentally, as well as a comparable model using a stochastic MC algorithm. The power of this research lies in the ability to compare and contrast experiment and model using each to develop the other.

\section{References}

$>1$ Kuharsky AL, Fogelson AL: Surface-mediated control of blood coagulation: the role of binding site densities and platelet deposition. Biophys J 2001;80:1050-1074.

$>2$ Doggett TA, Girdhar G, Lawshe A: Alterations in the intrinsic properties of the GPIbalphaVWF tether bond define the kinetics of the platelet-type von Willebrand disease mutation, Gly233Val. Blood 2003; 102:152-160.

-3 Laurenzi IJ, Diamond SL: Monte Carlo simulation of the heterotypic aggregation kinetics of platelets and neutrophils. Biophys J 1999;77: 1733-1746.

4 Hockin MF, Jones KC, Everse SJ, Mann KG A model for the stoichiometric regulation of blood coagulation. J Biol Chem 2002;277: 18322-18333.

5 Bungay SD, Gentry PA, Gentry RD: A mathematical model of lipid-mediated thrombin generation. Math Med Biol 2003;20:105129

6 Radhakrishnan K, Hindmarsh AC: Description and Use of LSODE, the Livermore Solver for Ordinary Differential Equations. LLNL report UCRL-ID-113855, Dec 1993

7 Gillespie DT: Exact stochastic simulation of coupled chemical reactions. J Phys Chem 1977; 81:2340-2361.

8 Lo K, Diamond SL: Blood coagulation kinetics: high throughput method for real-time reaction monitoring. Thromb Haemost 2004;92: 874-882.

-9 Eerkes A, Shou WZ, Naidong W: Liquid/liquid extraction using 96-well plate format in conjunction with hydrophilic interaction liquid chromatography - tandem mass spectrometry method for the analysis of fluconazole in human plasma. J Pharm Biomed Anal 2003;31: 917-928.

10 Xie F, Bruntlett CS, Zhu Y, Kissinger CB, Kissinger PT: Good preclinical bioanalytical chemistry requires proper sampling from laboratory animals: automation of blood and microdialysis sampling improves the productivity of LC/MSMS. Anal Sci 2003;19:479-485.
11 Sugimoto M, Matsui H, Mizuno T, Tsuji S, Miyata S, Matsumoto M, Matsuda M, Fujimura Y, Yoshioka A: Mural thrombus generation in type $2 \mathrm{~A}$ and $2 \mathrm{~B}$ von Willebrand disease under flow conditions. Blood 2003;101:915920.

12 Klein B, Faridi A, von Tempelhoff GF, Heilmann L, Mittermayer C, Rath W: A whole blood flow cytometric determination of platelet activation by unfractionated and low molecular weight heparin in vitro. Thromb Res 2003;108:291-296.

13 Gosalia DN, Diamond SL: Printing chemical libraries on microarrays for fluid phase nanoliter reactions. Proc Natl Acad Sci USA. 2003 100:8721-8726.

14 Gosalia DN, Salisbury CM, Ellman JA, Diamond SL: High throughput substrate specificity profiling of serine and cysteine proteases using solution-phase fluorogenic peptide microarrays. Mol Cell Proteomics 2005. Feb 10; [Epub ahead of print].

15 Gosalia DN, Salisbury CM, Maly DJ, Ellman JA, Diamond SL: Profiling serine protease substrate specificity with solution phase fluorogenic peptide microarrays. Proteomics 2005; in press.

16 Gailani D, Broze GJ: Factor XI activation in a revised model of blood coagulation. Science 1991;253:909-912.

17 Brummel-Ziedins KE, Pouliot RL, Mann KG: Thrombin generation: phenotypic quantitation. J Thromb Haemost 2004;2:281-288.

18 Butenas S, Brummel KE, Branda RF, Paradis SG, Mann KG: Mechanism of factor VIIa-dependent coagulation in hemophilia blood. Blood 2002;99:923-930.

19 Hemker HC, Giesen P, Al Dieri R, Regnault V, de Smedt E, Wagenvoord R, Lecompte T, Beguin S: Calibrated automated thrombin generation measurement in clotting plasma. Pathophysiol Haemost Thromb 2003;33:415.
20 Hemker HC, Giesen PL, Ramjee M, Wagenvoord $\mathrm{R}$, Beguin $\mathrm{S}$ : The thrombogram: monitoring thrombin generation in platelet-rich plasma. Thromb Haemost 2000;83:589-591.

21 Hemker HC, Giesen P, Al Dieri R, Regnault V, de Smed E, Wagenvoord R, Lecompte T, Beguin S: The calibrated automated thrombogram (CAT): a universal routine test for hyperand hypocoagulability. Pathophysiol Haemost Thromb 2002;32:249-253.

22 Matsumoto M, Nishimura T: Mersenne Twister: a 623-dimensionally equidistributed uniform pseudorandom number generator. ACM Transactions on Modeling and Computer Simulation 1998;7:3-30.

-23 Holmes MB, Schneider DJ, Hayes MG, Sobel BE, Mann KG: Novel, bedside, tissue factordependent clotting assay permits improved assessment of combination antithrombotic and antiplatelet therapy. Circulation 2000;102: 2051-2057.

-24 Kramer RM, Hession C, Johansen B, Hayes G, McGray P, Chow EP, Tizard R, Pepinsky RB: Structure and properties of a human non-pancreatic phospholipase A2. J Biol Chem 1989; 264:5768-5775.

25 Johnston GI, Pickett EB, McEver RP, George $\mathrm{JN}$ : Heterogeneity of platelet secretion in response to thrombin demonstrated by fluorescence flow cytometry. Blood 1987;69:14011403

26 Polgár J, Clemetson JM, Kehrel BE, Wiedemann M, Magnenat EM, Wells TN, Clemetson $\mathrm{KJ}$ : Platelet activation and signal transduction by convulxin, a C-type lectin from Crotalus durissus terrificus (tropical rattlesnake) venom via the p62/GPVI collagen receptor. J Biol Chem 1997;272:13576-13583.

27 Rauch U, Nemerson Y: Circulating tissue factor and thrombosis. Curr Opin Hematol 2000; 7:273-277.

28 Goel MS, Diamond SL: Factor VIIa-mediated tenase function on activated platelets under flow. J Thromb Haemost 2004;8:1402-1410.

-29 Coughlin SR: Thrombin signaling and protease-activated receptors. Nature 2000;407: 258-264 